\title{
Clinical benefit of remdesivir in rhesus macaques infected with SARS-CoV-2
}

https://doi.org/10.1038/s41586-020-2423-5

Received: 23 April 2020

Accepted: 2 June 2020

Published online: 9 June 2020

Check for updates

\author{
Brandi N. Williamson', Friederike Feldmann², Benjamin Schwarz ${ }^{3}$, Kimberly Meade-White', \\ Danielle P. Porter ${ }^{4}$, Jonathan Schulz', Neeltje van Doremalen', Ian Leighton ${ }^{3}$, \\ Claude Kwe Yinda', Lizzette Pérez-Pérez', Atsushi Okumura', Jamie Lovaglio², \\ Patrick W. Hanley' ${ }^{2}$, Greg Saturday², Catharine M. Bosio ${ }^{3}$, Sarah Anzick ${ }^{5}$, Kent Barbian ${ }^{5}$, \\ Tomas Cihlar ${ }^{4}$, Craig Martens ${ }^{5}$, Dana P. Scott ${ }^{2}$, Vincent J. Munster ${ }^{1} \&$ Emmie de Wit $^{1 凶}$
}

Effective therapies to treat coronavirus disease 2019 (COVID-19) are urgently needed. While many investigational, approved, and repurposed drugs have been suggested as potential treatments, preclinical data from animal models can guide the search for effective treatments by ruling out those that lack efficacy in vivo. Remdesivir (GS-5734) is a nucleotide analogue prodrug with broad antiviral activity ${ }^{1,2}$ that is currently being investigated in COVID-19 clinical trials and recently received Emergency Use Authorization from the US Food and Drug Administration ${ }^{3,4}$. In animal models, remdesivir was effective against infection with Middle East respiratory syndrome coronavirus (MERS-CoV) and severe acute respiratory syndrome coronavirus $(\mathrm{SARS}-\mathrm{CoV})^{2,5,6}$. In vitro, remdesivir inhibited replication of SARS-CoV-2,8. Here we investigate the efficacy of remdesivir in a rhesus macaque model of SARS-CoV-2 infection 9 . Unlike vehicle-treated animals, macaques treated with remdesivir did not show signs of respiratory disease; they also showed reduced pulmonary infiltrates on radiographs and reduced virus titres in bronchoalveolar lavages twelve hours after the first dose. Virus shedding from the upper respiratory tract was not reduced by remdesivir treatment. At necropsy, remdesivir-treated animals had lower lung viral loads and reduced lung damage. Thus, treatment with remdesivir initiated early during infection had a clinical benefit in rhesus macaques infected with SARS-CoV-2. Although the rhesus macaque model does not represent the severe disease observed in some patients with COVID-19, our data support the early initiation of remdesivir treatment in patients with COVID-19 to prevent progression to pneumonia.
We have recently established a rhesus macaque model of SARS-CoV-2 infection ${ }^{9}$. In this model, infected rhesus macaques develop mild to moderate, transient respiratory disease, with pulmonary infiltrates visible on radiographs and a shedding pattern similar to that observed in patients with COVID-19. The observed clinical signs and high viral loads enable the testing of the treatment efficacy of direct-acting antivirals in this model.

\section{Distribution of remdesivir to the lungs}

Two groups of six rhesus macaques were inoculated with SARS-CoV-2 strain nCoV-WA1-2020. Twelve hours after inoculation, one group was given $10 \mathrm{mg} \mathrm{kg}^{-1}$ intravenous remdesivir and the other group was treated with an equal volume of vehicle solution $\left(2 \mathrm{ml} \mathrm{kg}^{-1}\right)$. Treatment was continued $12 \mathrm{~h}$ after the first treatment and every $24 \mathrm{~h}$ thereafter with a dose of $5 \mathrm{mg} \mathrm{kg}^{-1}$ remdesivir or an equal volume of vehicle solution $\left(1 \mathrm{ml} \mathrm{kg}^{-1}\right)$. The concentration of remdesivir was determined in serum collected $12 \mathrm{~h}$ after the initial treatment and $24 \mathrm{~h}$ after subsequent doses (immediately before the next dose of treatment was administered). Remdesivir (prodrug GS-5734), its downstream alanine metabolite (GS-704277) and the parent nucleoside (GS-441524) were detected in serum from all remdesivir-treated animals (Extended Data Fig. 1a). Serum levels of the prodrug and downstream metabolites were consistent with previously published plasma levels of these compounds in healthy rhesus macaques, which showed a short systemic half-life for GS-5734 $(0.39 \mathrm{~h})$ resulting in transient conversion to the intermediate GS-704277 and persistence of the downstream GS-441524 product at higher plasma levels ${ }^{10}$.

Concentrations of the metabolite GS-441524 were measured in lung tissue collected from each lung lobe seven days post-inoculation (dpi) and $24 \mathrm{~h}$ after the last dose of remdesivir was administered; the metabolite was readily detectable in all remdesivir-treated animals. GS-441524 was generally distributed throughout all six lobes of the lung (Extended Data Fig. 1b). GS-704277 was not detected in lung tissue. Although the pharmacologically active metabolite of remdesivir is

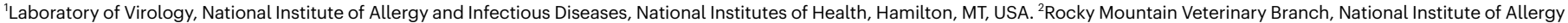
and Infectious Diseases, National Institutes of Health, Hamilton, MT, USA. ${ }^{3}$ Laboratory of Bacteriology, National Institute of Allergy and Infectious Diseases, National Institutes of Health,

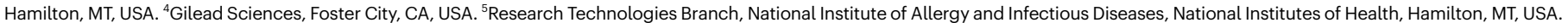
凶e-mail: emmie.dewit@nih.gov 

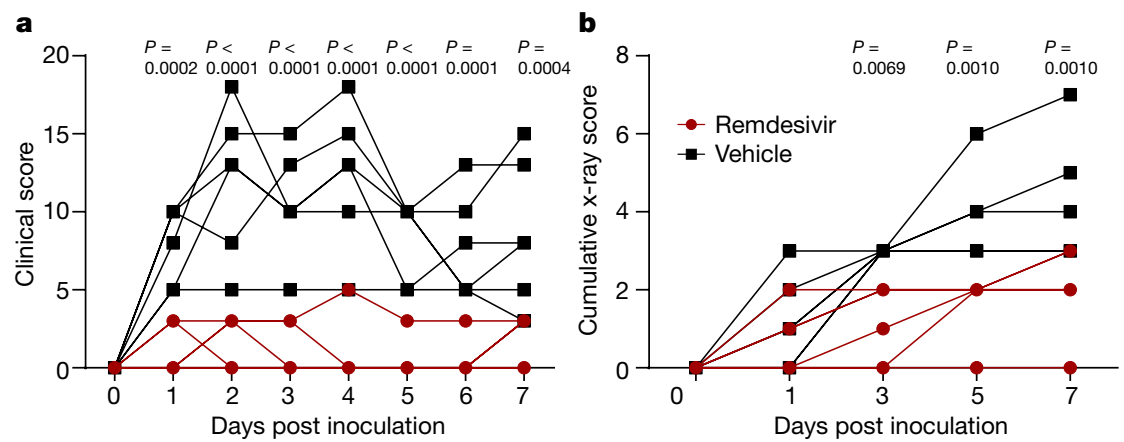

c

Remdesivir

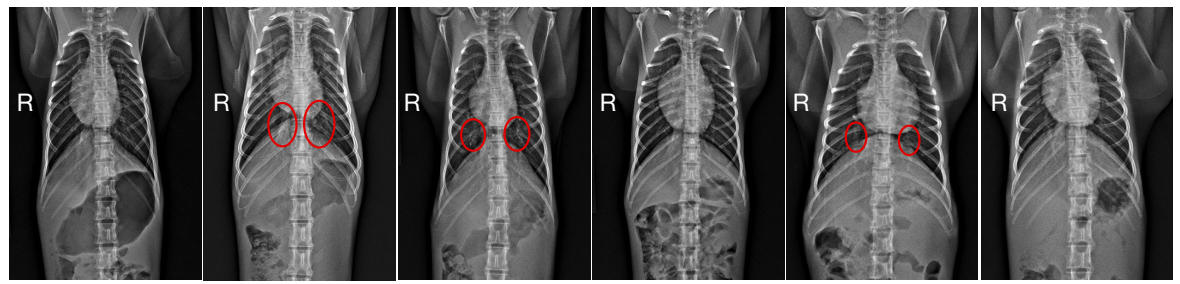

Vehicle
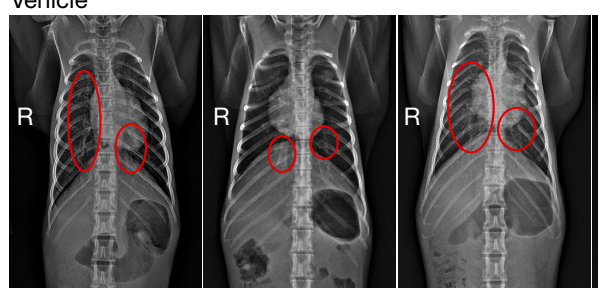

Fig. 1 Reduced respiratory disease in rhesus macaques infected with SARS-CoV-2 and treated with remdesivir. a, Daily clinical scores for animals infected with SARS-CoV-2 and treated with remdesivir (red circles, $n=6$ ) or vehicle solution (black squares, $n=6$ ). $\mathbf{b}$, Cumulative radiograph scores. Ventrodorsal and lateral radiographs were scored for the presence of pulmonary infiltrates by a clinical veterinarian according to a standard scoring system $(0$, normal; 1 , mild interstitial pulmonary infiltrates; 2 , moderate

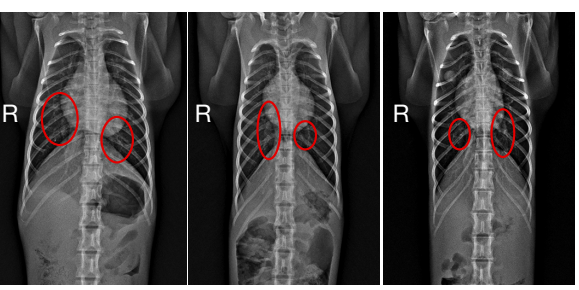

pulmonary infiltrates perhaps with partial cardiac border effacement and small areas of pulmonary consolidation; 3 , severe interstitial infiltrates, large areas of pulmonary consolidation, alveolar patterns and air bronchograms). Individual lobes were scored and scores per animal per day were totalled and displayed.c, Ventrodorsal radiographs for each animal taken on $7 \mathrm{dpi}$. Areas of pulmonary infiltration are circled. Statistical analysis was performed using a two-way ANOVA with Sidak's multiple comparisons test. the triphosphate of GS-441524, lung homogenate samples spiked with the triphosphate metabolite demonstrated rapid decay of the metabolite in this matrix (data not shown). GS-441524 levels were taken as a surrogate for tissue loading and suggest that the current dosing strategy delivered drug metabolites to the sites of SARS-CoV-2 replication in infected animals.

\section{Lack of respiratory disease}

After inoculation with SARS-CoV-2, the animals were assigned a daily clinical score based on a pre-established scoring sheet in a blinded fashion. Twelve hours after the first administration of remdesivir, clinical scores in remdesivir-treated animals were significantly lower
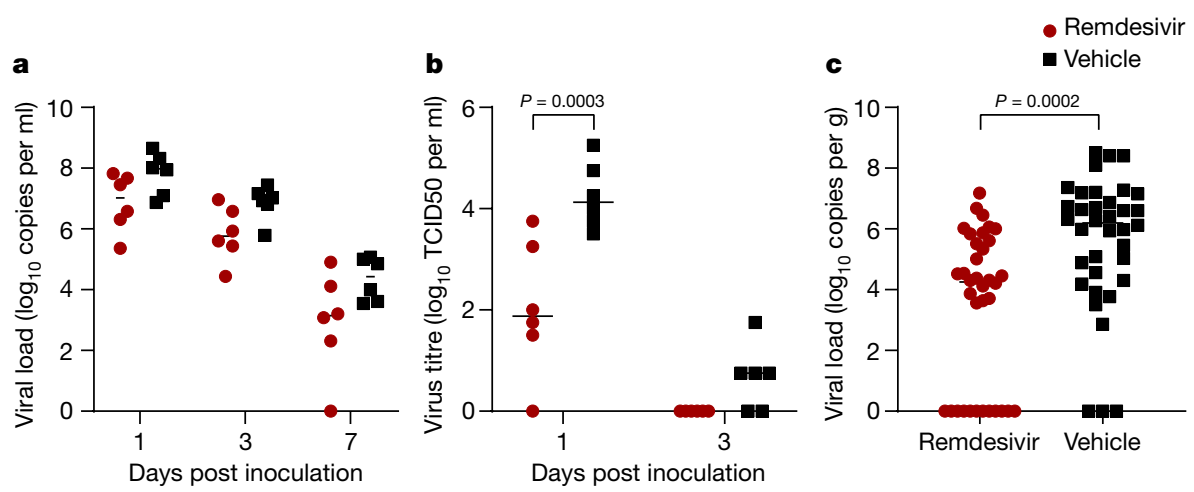

Fig. 2 | Viral loads and virus titres in BAL fluid and lung lobes. a, b, Viral loads (a) and infectious virus titres in BAL (b) collected from rhesus macaques infected with SARS-CoV-2 and treated with remdesivir $(n=6)$ or vehicle solution $(n=6)$. TCID50, 50\% tissue culture infectious dose. Statistical analysis was performed using a two-way ANOVA with Sidak's multiple comparisons test.

c, Viral loads in tissues collected from all six lung lobes at necropsy on $7 \mathrm{dpi}$ from rhesus macaques infected with SARS-CoV-2 and treated with remdesivir $(n=6)$ or vehicle solution $(n=6)$. Statistical analysis was performed using an unpaired $t$-test. Centre bars, median. 


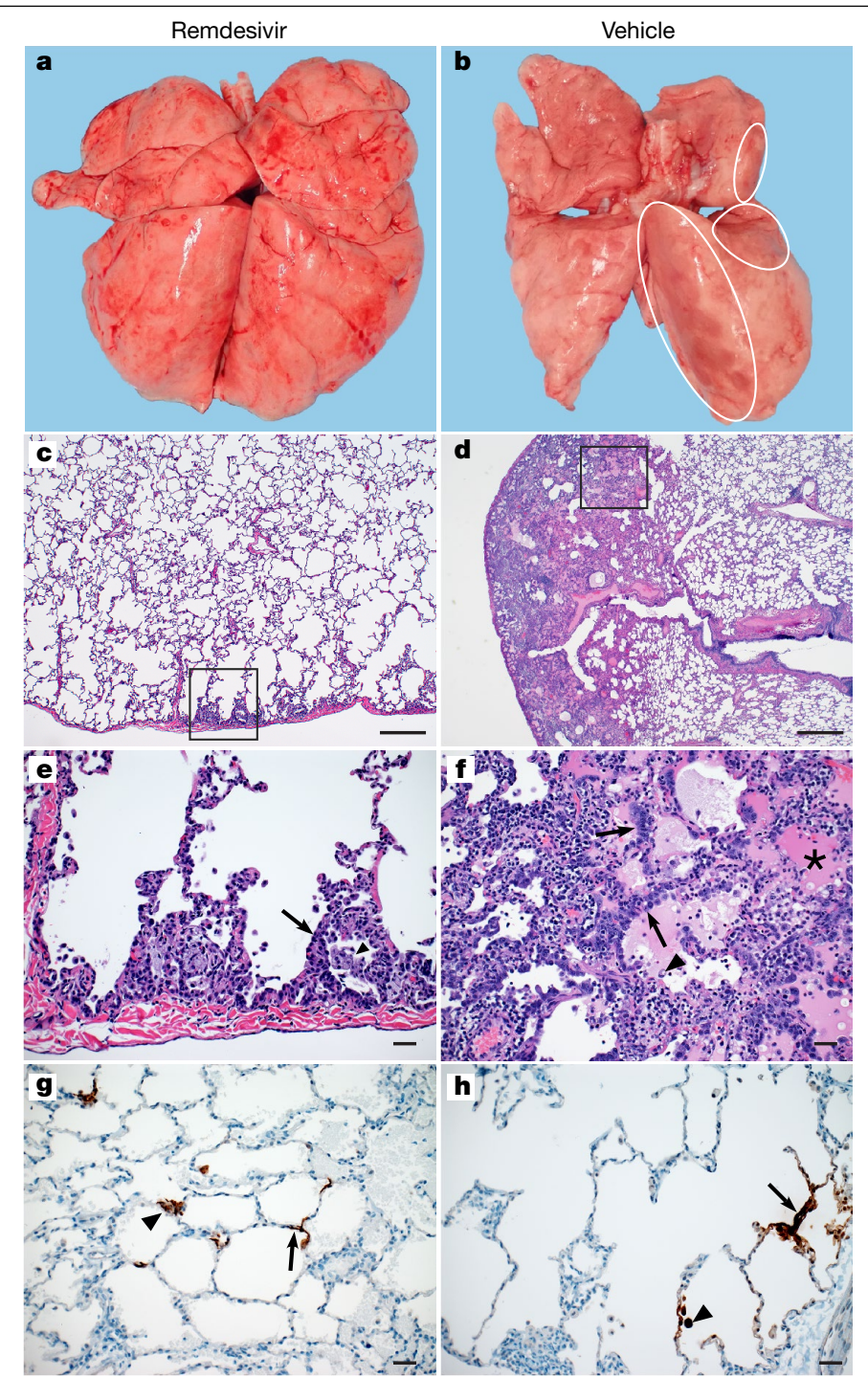

Fig. 3 | Changes to the lungs of rhesus macaques infected with SARS-CoV-2 and treated with remdesivir. Rhesus macaques infected with SARS-CoV-2 and treated with remdesivir (left, $n=6$ ) or vehicle solution (right, $n=6$ ) were euthanized on $7 \mathrm{dpi}$.a, $\mathbf{b}$, Representative dorsal views of lungs from a remdesivir-treated animal (a) and a vehicle-treated animal with focally extensive areas of consolidation (b, circles). Histological analysis was performed on three sections from six lung lobes from each of the six animals per treatment group and representative images were chosen for $\mathbf{c}-\mathbf{h}$. c, Minimal subpleural interstitial pneumonia (box) observed in three out of six remdesivir-treated animals. d, Moderate subpleural interstitial pneumonia with oedema (box) observed in five of six vehicle-treated animals. e, Boxed area from $\mathrm{c}$ with alveoli lined by type II pneumocytes (arrow) and alveolar spaces containing foamy macrophages (arrowhead).f, Boxed area from $\mathbf{d}$ with pulmonary interstitium expanded by oedema and moderate numbers of macrophages and neutrophils. Alveoli are lined by type II pneumocytes (arrows). Alveolar spaces are filled with oedema (asterisk) and small numbers of pulmonary macrophages (arrowhead). g, Viral antigen in type I pneumocytes (arrow) and type II pneumocytes (arrowhead) of a remdesivir-treated animal. $\mathbf{h}$, Viral antigen in type I pneumocytes (arrow) and macrophage (arrowhead) of a vehicle-treated animal. Scale bars: c, d, $200 \mu \mathrm{m}$; e-h, $20 \mu \mathrm{m}$.

than in control animals receiving vehicle solution. This difference in clinical score was maintained throughout the study (Fig. 1a). Only one of the six remdesivir-treated animals showed mild dyspnea, whereas tachypnea and dyspnea were observed in all vehicle-treated controls (Extended Data Table 1). Radiographic pulmonary infiltrates are one of the hallmarks of COVID-19 in humans. Radiographs taken on 0,1,3, 5 , and 7 dpi showed significantly less lung lobe involvement and less severe pulmonary infiltration in animals treated with remdesivir than in those treated with vehicle (Fig. 1b, c).

\section{Reduced virus replication in lungs}

On 1, 3 and $7 \mathrm{dpi}$, bronchoalveolar lavage (BAL) was performed as an indicator of virus replication in the lower respiratory tract. Although viral loads in BAL were reduced in animals treated with remdesivir, this difference was not statistically significant (Fig. 2a). However, $12 \mathrm{~h}$ after the first remdesivir treatment was administered, the infectious virus titre in BAL was about 100-fold lower in remdesivir-treated animals than in controls. By $3 \mathrm{dpi}$, infectious virus could no longer be detected in BAL from remdesivir-treated animals, whereas virus was still detected in BAL from four out of six control animals (Fig. 2b). Despite this reduction in virus replication in the lower respiratory tract, there was no reduction in viral load or infectious virus titre in nose, throat or rectal swabs collected from remdesivir-treated animals, except for a significant difference in virus titre in throat swabs collected on $1 \mathrm{dpi}$ and in viral loads in throat swabs collected on 4 dpi (Extended Data Fig. 2).

All animals were euthanized on $7 \mathrm{dpi}$. Tissue samples were collected from each lung lobe to compare virus replication between remdesivir-treated and vehicle-treated animals. In 10 out of 36 lung lobe samples collected from remdesivir-treated animals, viral RNA could not be detected, whereas this was the case in only 3 out of 36 lung lobes collected from control animals. In general, comparison across individual lung lobes in the two groups showed a lower geometric mean of viral RNA in the remdesivir-treated group (Extended Data Fig. 3a). Together, these data show that the viral load was significantly lower in lungs from remdesivir-treated animals than in those from vehicle-treated controls (Fig. 2c). Virus could be isolated from lung lobes of five out of six vehicle-treated control animals, but not from any of the lung tissue collected from remdesivir-treated animals. Although quantitative PCR with reverse transcription (qRT-PCR) showed that fewer tissues from other positions in the respiratory tract were positive for viral RNA in remdesivir-treated animals than in controls, these differences were not statistically significant (Extended Data Fig. 3b).

\section{Reduced pneumonia}

At necropsy on $7 \mathrm{dpi}$, lungs were assessed grossly for lesions. Gross lung lesions were observed in one out of six remdesivir-treated animals. By contrast, all six vehicle-treated control animals had visible lesions, resulting in a statistically significant difference in the area of the lungs affected by lesions (Fig. 3a, b, Extended Data Fig. 4a, b). This difference was also evident when calculating the lung weight-to-bodyweight ratio as an indicator of pneumonia; this ratio was significantly lower in remdesivir-treated than in vehicle-treated animals (Extended Data Fig. 4c). Histologically, remdesivir-treated animals had fewer and less severe lesions than did vehicle-treated controls. Histological lung lesions were absent in three out of six remdesivir-treated animals; the three remaining animals developed minimal pulmonary pathology. Lesions in these animals were characterized as widely separated, minimal, interstitial pneumonia frequently located in subpleural spaces (Fig. 3c, e). Five out six vehicle-treated animals developed multifocal, mild-to-moderate, interstitial pneumonia (Fig. 3d, f). We detected viral antigen in small numbers of type I and type II pneumocytes and alveolar macrophages in all animals, regardless of treatment (Fig. 3g, h).

\section{Absence of resistance mutations}

We successfully carried out deep sequencing on samples from all remdesivir-treated animals and vehicle-treated controls. Known mutations in the RNA-dependent RNA polymerase that confer resistance to 
remdesivir in coronaviruses ${ }^{11}$ were not detected in any of the samples tested (Supplementary Table 1).

\section{Discussion}

Remdesivir is, to our knowledge, the first antiviral treatment to show proven efficacy against SARS-CoV-2 in an animal model of COVID-19. Treatment of rhesus macaques infected with SARS-CoV-2 with remdesivir reduced clinical disease and damage to the lungs. The remdesivir dosing used in rhesus macaques is equivalent to that used in humans; however, owing to the acute nature of the disease in rhesus macaques, it is hard to directly translate the timing of treatment used to corresponding disease stages in humans. In our study, treatment was administered close to the peak of virus replication in the lungs as indicated by viral loads in bronchoalveolar lavages and the first effects of treatment on clinical signs and virus replication were observed within $12 \mathrm{~h}$. The efficacy of direct-acting antivirals against acute viral respiratory tract infections typically decreases with delays in treatment initation ${ }^{12}$. Thus, remdesivir treatment should be initiated as early as possible in patients with COVID-19 to achieve the maximum treatment effect.

Despite the lack of obvious respiratory signs and reduced virus replication in the lungs of remdesivir-treated animals, there was no reduction in virus shedding. This finding is very important for patient management, where a clinical improvement should not be interpreted as a lack of infectiousness. Although we have shown that remdesivir metabolites are found in the lower respiratory tract, drug levels in the upper respiratory tract have not been characterized and novel formulations with alternative routes of drug delivery should be considered to improve distribution to the upper respiratory tract, thereby reducing shedding and the potential transmission risk. However, as severe COVID-19 disease results from virus infection of the lungs, this organ is the main target of remdesivir treatment. The bioavailability and protective effect of remdesivir in the lungs of infected rhesus macaques support treatment of COVID-19 patients with remdesivir. Remdesivir treatment did not result in a clinical improvement in one clinical trial with patients with severe COVID-19 ${ }^{13}$; however, another clinical trial that involved more patients showed that remdesivir treatment resulted in a shorter time to improvement than in patients who received standard care only ${ }^{14}$. Our findings in rhesus macaques indicate that remdesivir treatment should be considered as early as clinically possible to prevent progression to pneumonia in patients with COVID-19.

\section{Online content}

Any methods, additional references, Nature Research reporting summaries, source data, extended data, supplementary information, acknowledgements, peer review information; details of author contributions and competing interests; and statements of data and code availability are available at https://doi.org/10.1038/s41586-020-2423-5.

1. Lo, M. K. et al. GS-5734 and its parent nucleoside analog inhibit Filo-, Pneumo-, and Paramyxoviruses. Sci. Rep. 7, 43395 (2017).

2. Sheahan, T. P. et al. Broad-spectrum antiviral GS-5734 inhibits both epidemic and zoonotic coronaviruses. Sci. Transl. Med. 9, eaal3653 (2017).

3. US National Library of Medicine Clinical Trials.gov (accessed 15 May 2020); https:// clinicaltrials.gov/ct2/results?cond=\&term=remdesivir\&cntry=\&state=\&city=\&dist=

4. US Food and Drug Administration. Coronavirus (COVID-19) Update: FDA Issues Emergency Use Authorization for Potential COVID-19 Treatment https://www.fda.gov/news-events/ press-announcements/coronavirus-covid-19-update-fda-issues-emergency-use-auth orization-potential-covid-19-treatment (2020).

5. de Wit, E. et al. Prophylactic and therapeutic remdesivir (GS-5734) treatment in the rhesus macaque model of MERS-CoV infection. Proc. Natl Acad. Sci. USA 117, 6771-6776 (2020).

6. Sheahan, T. P. et al. Comparative therapeutic efficacy of remdesivir and combination lopinavir, ritonavir, and interferon beta against MERS-CoV. Nat. Commun. 11, 222 (2020).

7. Choy, K. T. et al. Remdesivir, lopinavir, emetine, and homoharringtonine inhibit SARS-CoV-2 replication in vitro. Antiviral Res. 178, 104786 (2020).

8. Wang, M. et al. Remdesivir and chloroquine effectively inhibit the recently emerged novel coronavirus (2019-nCoV) in vitro. Cell Res. 30, 269-271 (2020).

9. Munster, V. J. et al. Respiratory disease in rhesus macaques inoculated with SARS-CoV-2 Nature https://doi.org/10.1038/s41586-020-2324-7 (2020).

10. Warren, T. K. et al. Therapeutic efficacy of the small molecule GS- 5734 against Ebola virus in rhesus monkeys. Nature 531, 381-385 (2016).

11. Agostini, M. L. et al. Coronavirus susceptibility to the antiviral remdesivir (GS-5734) is mediated by the viral polymerase and the proofreading exoribonuclease. MBio $\mathbf{9}$, e00221-18 (2018).

12. Sheahan, T. P. et al. An orally bioavailable broad-spectrum antiviral inhibits SARS-CoV-2 in human airway epithelial cell cultures and multiple coronaviruses in mice. Sci. Transl. Med. 12, eabb5883 (2020).

13. Wang, Y. et al. Remdesivir in adults with severe COVID-19: a randomised, double-blind, placebo-controlled, multicentre trial. Lancet 395, 1569-1578 (2020).

14. Fact Sheet for Health Care Providers Emergency Use Authorization (EUA) of Veklury (remdesivir) https://www.gilead.com/-/media/files/pdfs/remdesivir/ eua-fact-sheet-for-hcps_01may2020.pdf (Gilead Sciences, 2020).

Publisher's note Springer Nature remains neutral with regard to jurisdictional claims in published maps and institutional affiliations.

(c) This is a U.S. government work and not under copyright protection in the U.S.; foreign copyright protection may apply 2020 


\section{Methods}

\section{Ethics and biosafety statement}

All animal experiments were approved by the Institutional Animal Care and Use Committee of Rocky Mountain Laboratories, NIH and carried out by certified staff in an Association for Assessment and Accreditation of Laboratory Animal Care (AAALAC) International-accredited facility, according to the institution's guidelines for animal use, following the guidelines and basic principles in the NIH Guide for the Care and Use of Laboratory Animals, the Animal Welfare Act, United States Department of Agriculture and the United States Public Health Service Policy on Humane Care and Use of Laboratory Animals. Rhesus macaques were housed in adjacent individual primate cages, allowing social interactions, in a climate-controlled room with a fixed light-dark cycle ( $12 \mathrm{~h}$ light $-12 \mathrm{~h}$ dark). Animals were monitored at least twice daily throughout the experiment. Commercial monkey chow, treats, and fruit were provided twice daily by trained personnel. Water was available ad libitum. Environmental enrichment consisted of a variety of human interactions, manipulanda, commercial toys, videos, and music. The Institutional Biosafety Committee (IBC) approved work with infectious SARS-CoV-2 strains under BSL3 conditions. Sample inactivation was performed according to IBC-approved standard operating procedures for removal of specimens from high containment.

\section{Study design}

To evaluate the effect of remdesivir treatment on SARS-CoV-2 disease outcome, we used the recently established rhesus macaque model of SARS-CoV-2 infection that results in transient lower respiratory tract disease ${ }^{9}$. Since this is a model with little prior data, it was not possible to perform a power analysis to determine group size. The sample size was therefore based on experience with other nonhuman primate models of respiratory disease, mainly a rhesus macaque model of MERS-CoV where $n=6$ yielded statistical significance. Twelve animals were randomly assigned to two groups and inoculated as described previously with a total dose of $2.6 \times 10^{6}$ TCID50 (50\% tissue culture infectious dose) of SARS-CoV-2 strain nCoV-WA1-2020 via intranasal, oral, ocular and intratracheal routes. The efficacy of therapeutic remdesivir treatment was tested in two groups of six adult rhesus macaques (three males and three females each; $3.6-5.7 \mathrm{~kg}$ ). Owing to the acute nature of the SARS-CoV-2 model in rhesus macaques, therapeutic treatment was initiated $12 \mathrm{~h}$ after inoculation with SARS-CoV-2 and continued once daily for $6 \mathrm{dpi}$. One group of rhesus macaques was treated with a loading dose of $10 \mathrm{mg} / \mathrm{kg}$ remdesivir, followed by a daily maintenance dose of $5 \mathrm{mg} / \mathrm{kg}$. The other group of six animals served as infected controls and were administered an equal dose volume (that is, $2 \mathrm{ml} / \mathrm{kg}$ loading dose and $1 \mathrm{ml} / \mathrm{kg}$ thereafter) of vehicle solution (12\% sulfobutylether- $\beta$-cyclodextrin in water and hydrochloric acid, $\mathrm{pH} 3.5$ ) according to the same treatment schedule. This dosing scheme in rhesus macaques mimics the daily dosing tested in clinical studies involving patients with COVID-19 and results in similar systemic drug exposure. Treatment was delivered as an intravenous bolus injection (total dose delivered over approximately $5 \mathrm{~min}$ ) administered alternatingly in the left or right cephalic or saphenous veins. Although the remdesivir treatment course used here in rhesus macaques is shorter than the standard 10-day course in patients, this shorter treatment course was chosen to enable assessment of lung pathology at a time after inoculation when pulmonary infiltrates and interstitial pneumonia would still be present. Recent data from clinical trials have shown that a 5-day treatment course has a similar clinical benefit to a 10-day treatment course in patients with COVID-19 ${ }^{14}$.

The animals were observed twice daily for clinical signs of disease using a standardized scoring sheet as described previously ${ }^{9}$; the same person, who was blinded to the group assignment of the animals, assessed the animals throughout the study. The predetermined endpoint for this experiment was $7 \mathrm{dpi}$. Nose, throat and rectal swabs were collected daily during treatment administration. Clinical exams were performed on $0,1,3,5$, and $7 \mathrm{dpi}$ on anaesthetized animals. On exam days, clinical parameters such as bodyweight, temperature, pulse oximetry, blood pressure and respiration rate were collected, as well as dorsoventral and lateral chest radiographs. Radiographs were analysed by a clinical veterinarian blinded to the group assignment of the animals. On 1,3 and $7 \mathrm{dpi}$ a BAL was performed using $10 \mathrm{ml}$ sterile saline. After death on $7 \mathrm{dpi}$, necropsies were performed on the animals. The percentage of gross lung lesions were scored by a board-certified veterinary pathologist blinded to the group assignment of the animals and samples of the following tissues were collected: cervical lymph node, conjunctiva, nasal mucosa, oropharynx, tonsil, trachea, all lung lobes, mediastinal lymph node, right and left bronchus, heart, liver, spleen, kidney, stomach, duodenum, jejunum, ileum, caecum, colon, and urinary bladder. Histopathological analysis of tissue slides was performed by a board-certified veterinary pathologist blinded to the group assignment of the animals.

\section{Virus and cells}

SARS-CoV-2 isolate nCoV-WA1-2020 (MN985325.1) ${ }^{15}$ (Vero passage 3) was kindly provided by the Centers for Disease Control (CDC) and propagated once in Vero E6 cells in Dulbecco's modified Eagle's medium (DMEM, Sigma) supplemented with $2 \%$ fetal bovine serum (Gibco), $1 \mathrm{mM} \mathrm{L}$-glutamine (Gibco), $50 \mathrm{U} / \mathrm{ml}$ penicillin and $50 \mu \mathrm{g} / \mathrm{ml} \mathrm{streptomy-}$ cin (Gibco) (virus isolation medium). The virus stock used was $100 \%$ identical to the initial deposited GenBank sequence (MN985325.1) and no contaminants were detected. VeroE6 cells were maintained in DMEM supplemented with $10 \%$ fetal calf serum, $1 \mathrm{mM} \mathrm{L}$-glutamine, $50 \mathrm{U} / \mathrm{ml}$ penicillin and $50 \mu \mathrm{g} / \mathrm{ml}$ streptomycin.

\section{Remdesivir (GS-5734)}

Remdesivir (RDV; GS-5734) was manufactured at Gilead Sciences by the Department of Process Chemistry (Alberta, Canada) under Good Manufacturing Practice (GMP) conditions. Batch number 5734-BC-1P was solubilized in $12 \%$ sulfobutylether- $\beta$-cyclodextrin in water and matching vehicle solution was provided to $\mathrm{NIH}$.

\section{Liquid chromatography mass spectrometry (LC-MS)}

Tributylamine was purchased from Millipore Sigma. LC-MS grade water, acetone, methanol, isopropanol and acetic acid were purchased through Fisher Scientific. All synthetic standards for molecular analysis were provided by Gilead Sciences Inc. Serum and cleared lung homogenates were gamma-irradiated ( $2 \mathrm{MRad}$ ) to inactivate infectious virus potentially present in these samples before analysis. Samples were prepared for small molecule analysis by diluting a $50-\mu \mathrm{l}$ aliquot of either serum or clarified lung homogenate with $950 \mu \mathrm{l}$ of $50 \%$ acetone, 35\% methanol, $15 \%$ water ( $\mathrm{v} / \mathrm{v})$ on ice. Samples were incubated at room temperature for $15 \mathrm{~min}$ and then centrifuged at $16,000 \mathrm{~g}$ for $5 \mathrm{~min}$. The clarified supernatants $(850 \mu \mathrm{l})$ were recovered and taken to dryness in a Savant DNA120 SpeedVac concentrator (Thermo Fisher). Samples were resuspended in $100 \mu$ l of $50 \%$ methanol, $50 \%$ water $(\mathrm{v} / \mathrm{v})$ and centrifuged as before. The supernatant was taken to a sample vial for LC-MS analysis. Samples were separated using an ion-pairing liquid chromatography strategy on a Sciex ExionLC AC system. Samples were injected onto a Waters Atlantis T3 column $(100 \AA, 3 \mu \mathrm{m}, 3 \mathrm{~mm} \times 100 \mathrm{~mm})$ and eluted using a binary gradient from $5 \mathrm{mM}$ tributylamine, $5 \mathrm{mM}$ acetic acid in $2 \%$ isopropanol, $5 \%$ methanol, $93 \%$ water (v/v) to $100 \%$ isopropanol over $5.5 \mathrm{~min}$. Analytes were measured using a Sciex 5500 QTRAP mass spectrometer in negative mode. Multiple reaction monitoring was performed using two signal pairs for each analyte and signal fidelity was confirmed by collecting triggered product ion spectra and comparing back to spectra of synthetically pure standards.

All analytes were quantified against an eight-point calibration curve of the respective synthetic standard prepared in the target matrix (that is, serum or cleared lung homogenate) and processed in the same 
manner as experimental samples. Limit of quantification (LOQ) was approximated at a signal to noise of 10 . The LOQs for the measured molecules in each matrix were $5 \mathrm{nM}$ for GS-441524 in both lung homogenate and serum, $1 \mathrm{nM}$ for GS-704277 in both lung homogenate and serum and $0.08 \mathrm{nM}$ for GS-5734 in serum. Instability of GS-5734 and the tri-phosphorylated nucleotide metabolite in the lung homogenate during tissue lysis prevented detection of these metabolites in the lung tissue.

\section{Quantitative PCR}

RNA was extracted from swabs and BAL using the QiaAmp Viral RNA kit (Qiagen) according to the manufacturer's instructions. Tissues (30 mg) were homogenized in RLT buffer and RNA was extracted using the RNeasy kit (Qiagen) according to the manufacturer's instructions. For detection of viral RNA, $5 \mu$ RNA was used in a one-step real-time RT-PCR E assay ${ }^{16}$ using the Rotor-Gene probe kit (Qiagen) according to the manufacturer's instructions. In each run, standard dilutions of RNA standards counted by droplet digital PCR were run in parallel, to calculate copy numbers in the samples.

\section{Virus titration}

Virus titrations were performed by end-point titration in Vero E6 cells. Tissue was homogenized in $1 \mathrm{ml}$ DMEM using a TissueLyser (Qiagen). Cells were inoculated with tenfold serial dilutions of swab and BAL samples. Virus isolation was performed on lung tissues by homogenizing the tissue in $1 \mathrm{ml}$ DMEM and inoculating Vero E6 cells in a 24-well plate with $250 \mu$ l cleared homogenate and a 1:10 dilution thereof. One hour after inoculation of cells, the inoculum was removed and replaced with $100 \mu \mathrm{l}$ (virus titration) or $500 \mu \mathrm{l}$ virus isolation medium. Six days after inoculation, CPE was scored and the TCID50 was calculated.

\section{Histopathology and immunohistochemistry}

Histopathology and immunohistochemistry were performed on rhesus macaque tissues. After fixation for a minimum of 7 days in $10 \%$ neutral-buffered formalin and embedding in paraffin, tissue sections were stained with haematoxylin and eosin (H\&E). To detect SARS-CoV-2 antigen, immunohistochemistry was performed using a custom-made rabbit antiserum against SARS-CoV-2 $\mathrm{N}$ at a 1:1,000 dilution. Stained slides were analysed by a board-certified veterinary pathologist.

\section{Next generation sequencing of viral RNA}

Viral RNA was extracted as described above. cDNAs were prepared as described, with minor modifications ${ }^{17}$. In brief, 3-12 $\mu$ l of extracted RNA was depleted of rRNA using Ribo-Zero Gold H/M/R (Illumina) and then reverse-transcribed using random hexamers and SuperScript IV (ThermoFisher Scientific). Following RNaseH treatment, second strand synthesis was performed using Klenow fragment (New England Biolabs) and resulting double-stranded cDNAs were treated with a combined mixture of RiboShredder RNase Blend (Lucigen) and RNase, DNase-free, high conc (Roche Diagnostics, Indianapolis, IN) and then purified using Ampure XP bead purification (Beckman Coulter). Kapa's HyperPlus library preparation kit (Roche Sequencing Solutions) was used to prepare sequencing libraries from the double-stranded cDNAs. To facilitate multiplexing, adaptor ligation was performed with KAPA Unique Dual-Indexed Adapters and samples were enriched for adaptor-ligated product using KAPA HiFi HotStart Ready mix and seven PCR amplification cycles, according to the manufacturer's manual. Pools consisting of eight sample libraries were used for hybrid-capture virus enrichment using myBaits Expert Virus SARS-CoV-2 panel and following the manufacturer's manual, version 4.01, with 14 cycles of post-capture PCR amplification (Arbor Biosciences). Purified, enriched libraries were quantified using Kapa Library Quantification kit (Roche Sequencing Solutions) and sequenced as $2 \times 150$-base pair reads on the Illumina NextSeq 550 instrument (Illumina).

Raw fastq reads were trimmed of Illumina adaptor sequences using cutadapt version $1.12^{18}$ and then trimmed and filtered for quality using the FASTX-Toolkit (Hannon Lab). Remaining reads were mapped to the SARS-CoV-2 2019-nCoV/USA-WA1/2020 genome (MN985325.1) using Bowtie2 version 2.2.9 ${ }^{19}$ with parameters --local--no-mixed-X1500. PCR duplicates were removed using picard MarkDuplicates (Broad Institute) and variants were called using GATK HaplotypeCaller version 4.1.2.0 ${ }^{20}$ with parameter -ploidy 2. Variants were filtered for QUAL $>1000$ and DP $>20$ using bcftools.

\section{Statistical analysis}

Statistical analyses were performed using GraphPad Prism software version 8.2.1.

\section{Reporting summary}

Further information on research design is available in the Nature Research Reporting Summary linked to this paper.

\section{Data availability}

All data included in this manuscript have been deposited in Figshare (https://doi.org/10.35092/yhjc.12111570). Sequences have been deposited in NCBI under BioProject accession number PRJNA632475.

15. Harcourt, J. et al. Severe acute respiratory syndrome coronavirus 2 from patient with coronavirus disease, United States. Emerg. Infect. Dis. 26, 1266-1273 (2020).

16. Corman, V. M. et al. Detection of 2019 novel coronavirus (2019-nCoV) by real-time RT-PCR. Euro Surveill. 25, 2000045 (2020).

17. Briese, $T$. et al. Virome capture sequencing enables sensitive viral diagnosis and comprehensive virome analysis. MBio 6, e01491-15 (2015).

18. Martin M. Cutadapt removes adapter sequences from high-throughput sequencing reads. EMBnet.journal 17, 10-12 (2011).

19. Langmead, B. \& Salzberg, S. L. Fast gapped-read alignment with Bowtie 2. Nat. Methods 9, 357-359 (2012).

20. McKenna, A. et al. The Genome Analysis Toolkit: a MapReduce framework for analyzing next-generation DNA sequencing data. Genome Res. 20, 1297-1303 (2010).

Acknowledgements We thank E. Bunyan for preparing remdesivir; D. Babusis for providing synthetic standards for molecular analysis; A. Mora for preparing figures; T. Thomas, R. Rosenke and D. Long for assistance with histology; M. Holbrook and T. Bushmaker for technical assistance; and RMVB staff for animal care. This study was supported by the Intramural Research Program of NIAID, NIH.

Author contributions D.P.P., T.C., V.J.M. and E.d.W. designed the study; B.N.W., F.F., B.S., K.M.-W., J.S., N.v.D., I.L., C.K.Y., L.P.-P., A.O., J.L., P.W.H., G.S., S.A., K.B., C.M., D.P.S., V.L.M. and E.d.W. acquired and analysed the data; B.N.W., F.F., B.S., D.P.P., N.v.D., C.K.Y., A.O., J.L., P.W.H., G.S., C.M.B., S.A., K.B., T.C., C.M., D.P.S., V.J.M. and E.d.W. interpreted the data; and E.d.W. wrote the manuscript. All authors approved the submitted version of the manuscript.

Competing interests D.P.P. and T.C. are employees of Gilead Sciences and own company stock. The remaining authors report no competing interests.

Additional information

Supplementary information is available for this paper at https://doi.org/10.1038/s41586-0202423-5.

Correspondence and requests for materials should be addressed to E.d.W.

Peer review information Nature thanks Wolfgang Baumgärtner, Stanley Pearlman and the other, anonymous, reviewer(s) for their contribution to the peer review of this work. Reprints and permissions information is available at http://www.nature.com/reprints. 

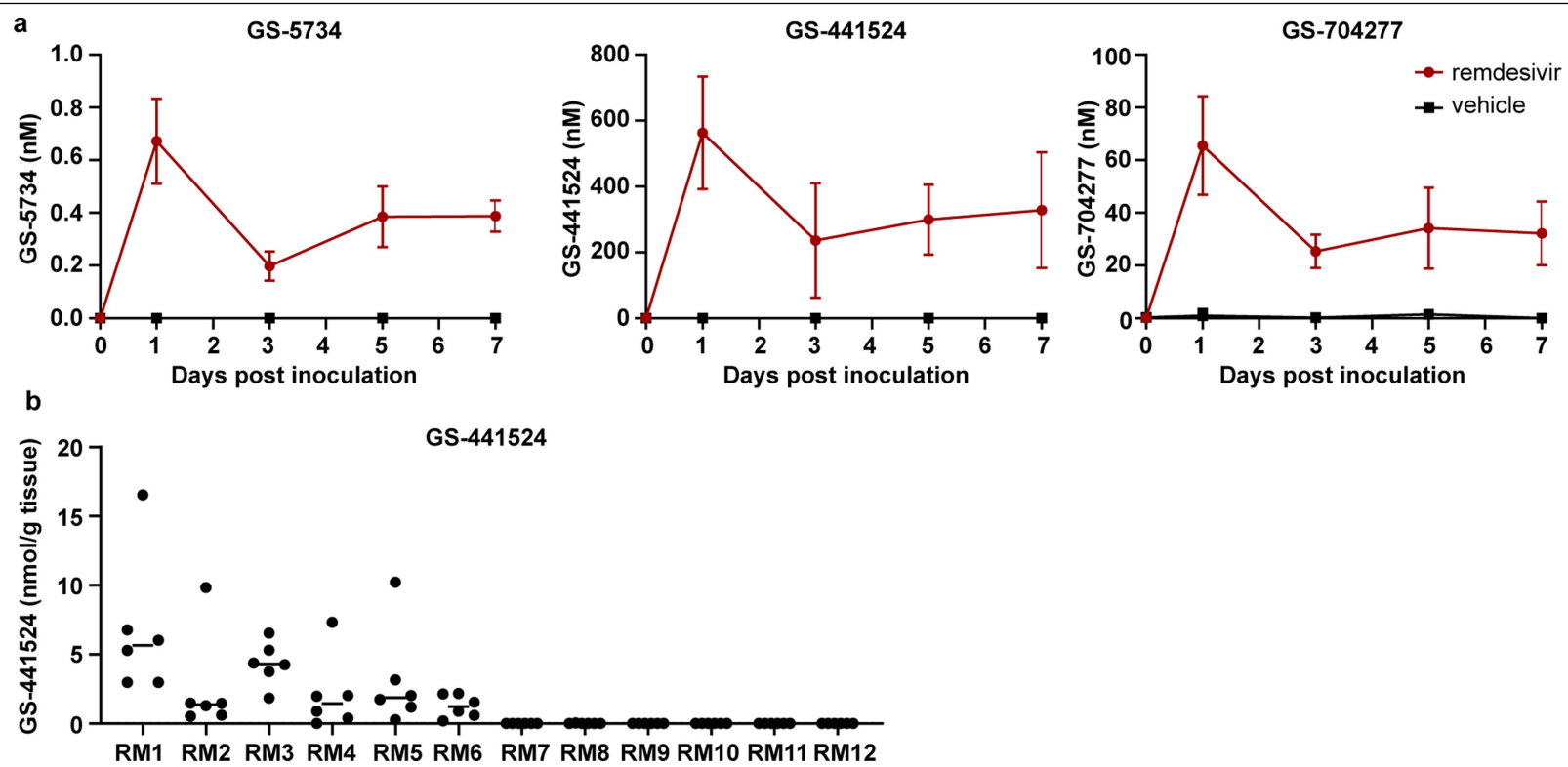

Extended Data Fig. 1 | Concentration of remdesivir prodrug and metabolites measured in serum and lung homogenates of rhesus macaques infected with SARS-CoV-2. Two groups of six rhesus macaques were inoculated with SARS-CoV-2 strain nCoV-WA1-2020. Twelve hours post inoculation, one group was administered $10 \mathrm{mg} / \mathrm{kg}$ intravenous remdesivir and the other group was treated with an equal volume of vehicle solution $(2 \mathrm{ml} / \mathrm{kg})$. Treatment was continued $12 \mathrm{hrs}$ after the first treatment, and every $24 \mathrm{~h}$ thereafter with a dose of $5 \mathrm{mg} / \mathrm{kg}$ remdesivir or equal volume of vehicle solution $(1 \mathrm{ml} / \mathrm{kg})$. a, Serum concentration of remdesivir prodrug GS-5734, the dephosphorylated nucleoside product GS-441524 and the intermediate alanine metabolite GS-704277 over time as measured by LCMS for all animals $(n=12)$ in the study. Mean and standard deviation are shown. b, Concentration of GS-441524 homogenized lung tissue collected from all six lung lobes from each animal ( $n=12)$ on $7 \mathrm{dpi}, 24 \mathrm{~h}$ after the last remdesivir treatment was administered. Each dot represents the concentration of GS-441524 in one lung lobe. The centre bar represents the median. 
a

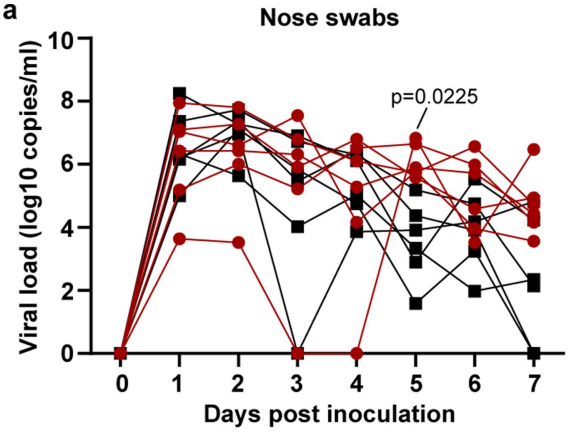

b

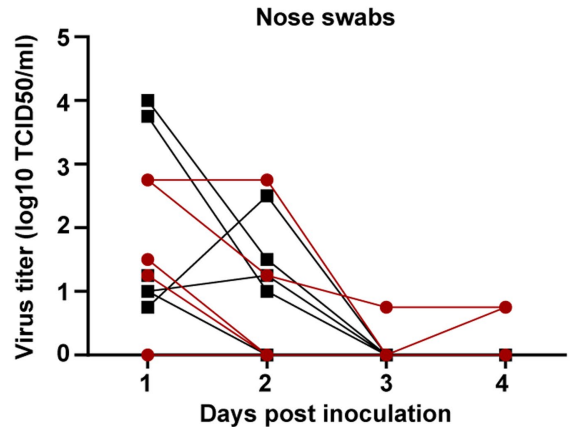

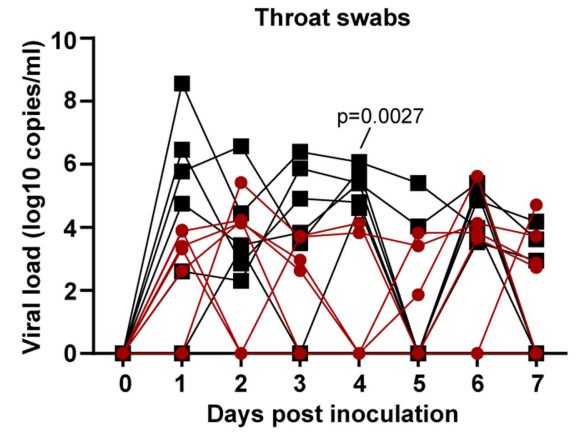

Days post inoculation

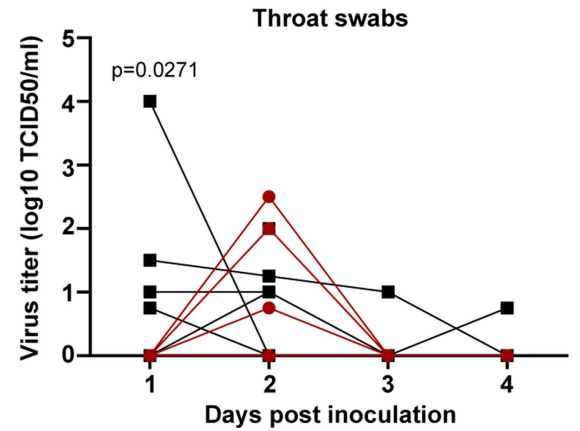

Rectal swabs
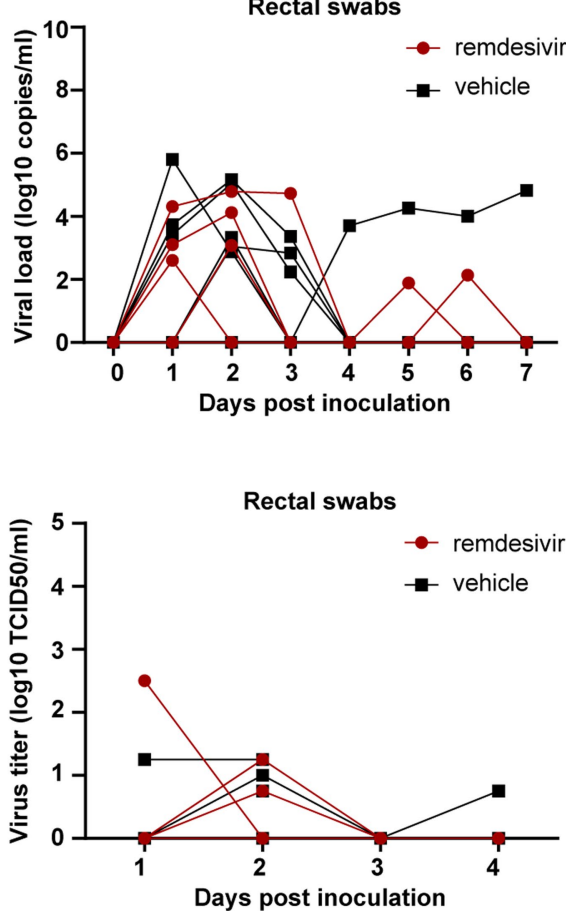

Extended Data Fig. 2 | Viral loads and virus titres in swabs collected from rhesus macaques infected with SARS-CoV-2 and treated with remdesivir. a, Viral loads; b, infectious virus titers in nose, throat and rectal swabs collected daily from animals treated with remdesivir $(n=6)$ or vehicle solution $(n=6)$. Statistical analysis was performed using a 2-way ANOVA with Sidak's multiple comparisons test. 


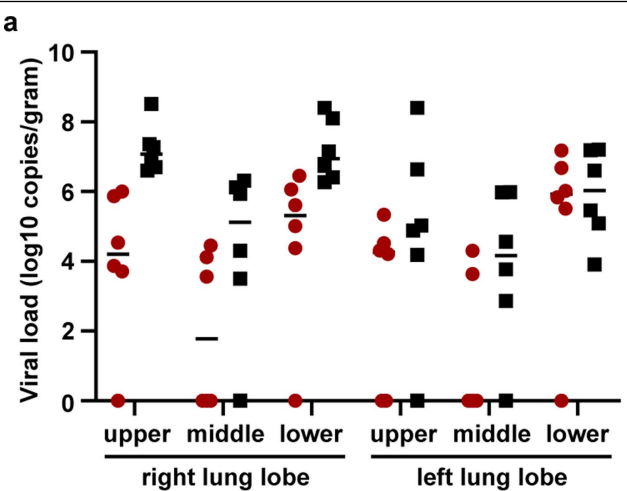

b

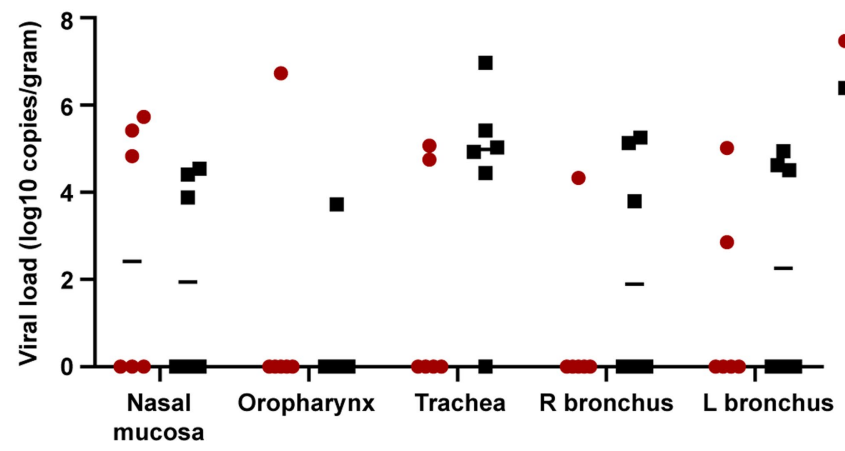

Extended Data Fig. 3 | Viral loads in tissues collected from the respiratory tract on $7 \mathrm{dpi}$. a, Viral loads in all six lung lobes collected from rhesus macaques infected with SARS-CoV-2 and treated with remdesivir $(n=6)$ or vehicle solution $(n=6)$, stratified per lung lobe. $\mathbf{b}$, Viral loads in other tissues collected throughout the respiratory tract on $7 \mathrm{dpi}$. The centre bar represents the median. remdesivir

vehicle 


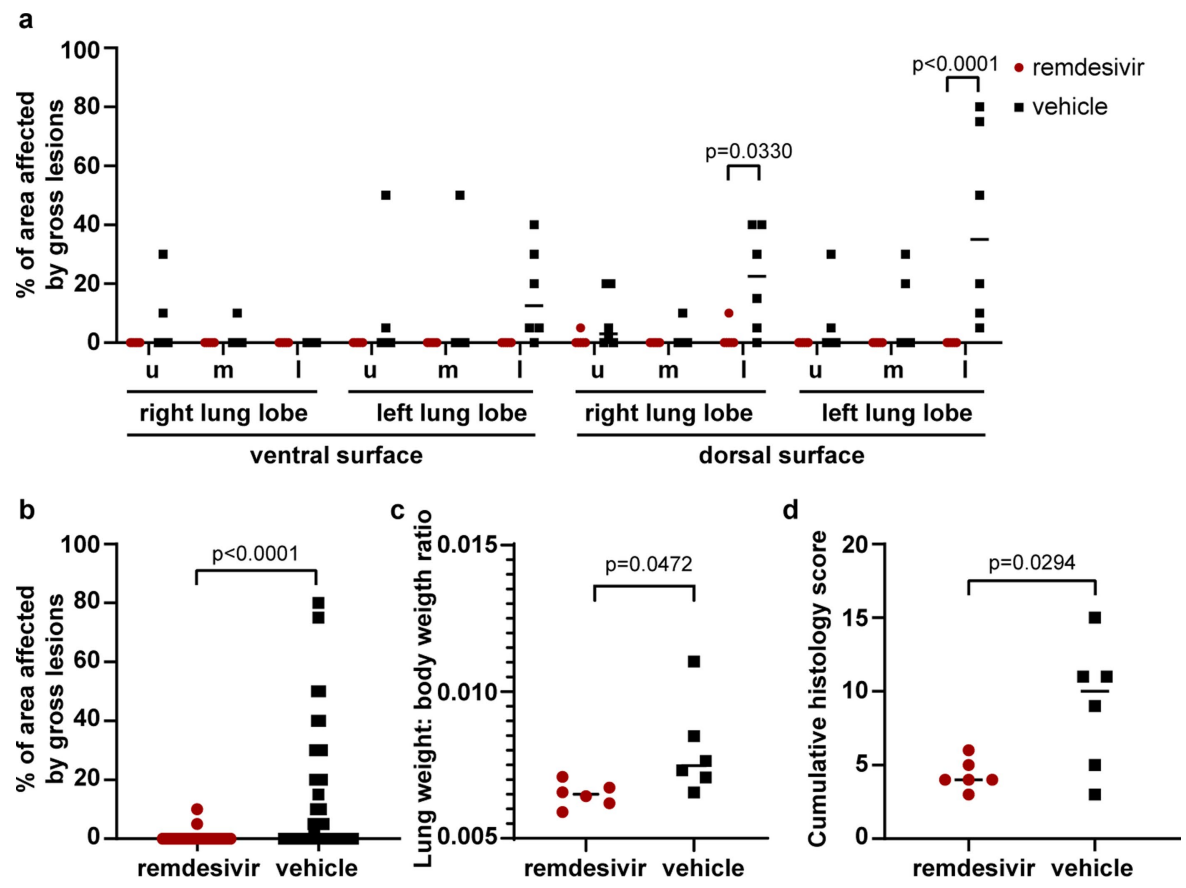

Extended Data Fig. 4 | Pathological changes in lungs of rhesus macaques infected with SARS-CoV-2 and treated with remdesivir. Rhesus macaques infected with SARS-CoV-2 and treated with remdesivir $(n=6)$ or vehicle solution $(n=6)$ were euthanized on $7 \mathrm{dpi}$. a, The area of each individual lung lobe affected by gross lesions as scored by a veterinary pathologist blinded to group assignment of the animals. b, All data from panel a combined. c, Lung weight: bodyweight ratio as an indicator of pulmonary oedema.d, Cumulative histology score. Each lung lobe was scored for the presence of histological lung lesions on a predetermined scale $(0-4)$; these values were combined per animal and graphed. Data in panel a were analysed using a 2-way ANOVA with Sidak's multiple comparisons test; data in b-d were analysed using a two-tailed, unpaired $t$-test. The centre bar represents the median. 
Extended Data Table 1 | Clinical and pathological observations in rhesus macaques inoculated with SARS-CoV-2 and treated with remdesivir

\begin{tabular}{|c|c|c|c|}
\hline Treatment & Animal & Clinical observations & Observations at necropsy \\
\hline \multirow[t]{6}{*}{ Remdesivir } & RM1 & Slightly decreased appetite & Mediastinal lymph nodes enlarged \\
\hline & $\mathrm{RM} 2$ & Slightly decreased appetite & None \\
\hline & RM3 & Slightly decreased appetite, pale appearance & Mediastinal lymph nodes enlarged \\
\hline & RM4 & Slightly decreased appetite, slightly dehydrated & Mediastinal lymph nodes enlarged \\
\hline & RM5 & Slightly decreased appetite & Mediastinal lymph nodes enlarged \\
\hline & RM6 & Mild dyspnea, pale appearance & $\begin{array}{l}\text { Gross lung lesions; mediastinal lymph } \\
\text { nodes enlarged }\end{array}$ \\
\hline \multirow[t]{6}{*}{ Vehicle solution } & RM7 & $\begin{array}{l}\text { Piloerection, hunched posture, tachypnea, dyspnea, decreased } \\
\text { appetite }\end{array}$ & $\begin{array}{l}\text { Gross lung lesions; mediastinal lymph } \\
\text { nodes enlarged; focal hemorrhage in } \\
\text { colon }\end{array}$ \\
\hline & RM8 & $\begin{array}{l}\text { Piloerection, hunched posture, tachypnea, dyspnea, decreased } \\
\text { appetite }\end{array}$ & $\begin{array}{l}\text { Gross lung lesions; mediastinal lymph } \\
\text { nodes enlarged }\end{array}$ \\
\hline & RM9 & $\begin{array}{l}\text { Piloerection, hunched posture, tachypnea, dyspnea, decreased } \\
\text { appetite }\end{array}$ & $\begin{array}{l}\text { Gross lung lesions; mediastinal lymph } \\
\text { nodes enlarged }\end{array}$ \\
\hline & RM10 & Tachypnea, dyspnea, pale appearance, slightly dehydrated & $\begin{array}{l}\text { Gross lung lesions; mediastinal lymph } \\
\text { nodes enlarged }\end{array}$ \\
\hline & RM11 & $\begin{array}{l}\text { Piloerection, tachypnea, dyspnea, decreased appetite, pale } \\
\text { appearance }\end{array}$ & $\begin{array}{l}\text { Gross lung lesions; mediastinal lymph } \\
\text { nodes enlarged }\end{array}$ \\
\hline & RM12 & Piloerection, tachypnea, dyspnea, decreased appetite & $\begin{array}{l}\text { Gross lung lesions; mediastinal lymph } \\
\text { nodes enlarged; } \sim 5 \mathrm{ml} \text { fluid in } \\
\text { peritoneum }\end{array}$ \\
\hline
\end{tabular}




\section{Reporting Summary}

Nature Research wishes to improve the reproducibility of the work that we publish. This form provides structure for consistency and transparency in reporting. For further information on Nature Research policies, seeAuthors \& Referees and theEditorial Policy Checklist.

\section{Statistics}

For all statistical analyses, confirm that the following items are present in the figure legend, table legend, main text, or Methods section.

$\mathrm{n} / \mathrm{a} \mid$ Confirmed

$\square \quad \boldsymbol{x}$ The exact sample size $(n)$ for each experimental group/condition, given as a discrete number and unit of measurement

$\mathbf{x} \square$ A statement on whether measurements were taken from distinct samples or whether the same sample was measured repeatedly

$\boldsymbol{x}$ The statistical test(s) used AND whether they are one- or two-sided

X Only common tests should be described solely by name; describe more complex techniques in the Methods section.

\begin{tabular}{|l|l}
$\boldsymbol{x}$ \\
\end{tabular} A description of all covariates tested

X A description of any assumptions or corrections, such as tests of normality and adjustment for multiple comparisons

$\boldsymbol{x} \square$ A full description of the statistical parameters including central tendency (e.g. means) or other basic estimates (e.g. regression coefficient)

$x \square$ AND variation (e.g. standard deviation) or associated estimates of uncertainty (e.g. confidence intervals)

$\mathbf{x} \square$ For null hypothesis testing, the test statistic (e.g. $F, t, r$ ) with confidence intervals, effect sizes, degrees of freedom and $P$ value noted

$\mathbf{x} \square$ Give $P$ values as exact values whenever suitable.

$\mathbf{x} \square$ For Bayesian analysis, information on the choice of priors and Markov chain Monte Carlo settings

$\boldsymbol{x} \square$ For hierarchical and complex designs, identification of the appropriate level for tests and full reporting of outcomes

$\boldsymbol{x} \square$ Estimates of effect sizes (e.g. Cohen's $d$, Pearson's $r$ ), indicating how they were calculated

Our web collection on statistics for biologists contains articles on many of the points above.

\section{Software and code}

Policy information about availability of computer code

Data collection Sequence analysis software used: Cutadapt version 1.12; FASTX-Toolkit; Bowtie2 version 2.2.9; picard MarkDuplicates; GATK HaplotypeCaller version 4.1.2.0; bcftools; GATK version 3 DepthOfCoverage tool

Data analysis Data were analyzed using Graphad Prism 8.2

For manuscripts utilizing custom algorithms or software that are central to the research but not yet described in published literature, software must be made available to editors/reviewers. We strongly encourage code deposition in a community repository (e.g. GitHub). See the Nature Research guidelines for submitting code \& software for further information.

\section{Data}

Policy information about availability of data

All manuscripts must include a data availability statement. This statement should provide the following information, where applicable:

- Accession codes, unique identifiers, or web links for publicly available datasets

- A list of figures that have associated raw data

- A description of any restrictions on data availability

Data have been deposited in Figshare: https://doi.org/10.1101/2020.04.15.043166

Sequences have been deposited in NCBI, BioProject accession number PRJNA632475

\section{Field-specific reporting}

Please select the one below that is the best fit for your research. If you are not sure, read the appropriate sections before making your selection. 


\section{Life sciences study design}

All studies must disclose on these points even when the disclosure is negative.

Sample size Since this is a model with little prior data, it was not possible to perform a power analysis. The sample size was based on experience with other nonhuman primate models of respiratory disease, mainly a rhesus macaque model of MERS-CoV where $n=6$ yielded statistical significance.

Data exclusions No data were excluded.

Replication Lung histology: for each animal ( $n=6$ per group), 3 sections were evaluated from all 6 lung lobes.

Radiographs: Three chest radiographs were taken from each animal at each clinical exam: right-lateral, left-lateral and ventro-dorsal; only the ventro-dorsal radiograph is shown.

Randomization Animals were randomly assigned to the group administered remdesivir or vehicle solution.

Blinding The following tasks were performed by researchers blinded to group assignment: daily clinical scoring; analysis of radiographs; histopathology

\section{Reporting for specific materials, systems and methods}

We require information from authors about some types of materials, experimental systems and methods used in many studies. Here, indicate whether each material, system or method listed is relevant to your study. If you are not sure if a list item applies to your research, read the appropriate section before selecting a response.

Materials \& experimental systems

\begin{tabular}{|c|c|c|}
\hline$n / a$ & \multicolumn{2}{|c|}{ Involved in the study } \\
\hline L & $x$ & Antibodies \\
\hline 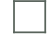 & $x$ & Eukaryotic cell lines \\
\hline$x$ & \begin{tabular}{|r} 
\\
\end{tabular} & Palaeontology \\
\hline & $x$ & Animals and other organisms \\
\hline$x$ & \begin{tabular}{|r} 
\\
\end{tabular} & Human research participants \\
\hline$x$ & & Clinical data \\
\hline
\end{tabular}

Methods

$\mathrm{n} / \mathrm{a}$ Involved in the study

$\boldsymbol{x} \square$ ChIP-seq

$\mathbf{x} \square$ Flow cytometry

$\mathbf{x} \square$ MRI-based neuroimaging

\section{Antibodies}

Antibodies used

Validation
Custom-ordered anti-SARS-CoV-2 nucleocapsid antibody; generated in rabbits by GenScript. Since this is a custom order there is no catalog number for this antibody.

Validation of cross-reactivity of the SARS-CoV-2 custom antibody in IHC was done in-house by embedding SARS-CoV-2 infected Vero cells in histogel and producing and staining histology slides; this was then confirmed by staining known SARS-CoV-2-postive lung tissue as well as negative control tissue.

\section{Eukaryotic cell lines}

\section{Policy information about cell lines}

Cell line source(s)

VeroE6: Ralph Baric, University of North Carolina, Chapel Hill, USA

Authentication

Not authenticated in-house.

Mycoplasma contamination

Mycoplasma testing confirmed negative at regular intervals.

Commonly misidentified lines (See ICLAC register)

No commonly misidentified cell lines were used.

\section{Animals and other organisms}

Policy information about studies involving animals; ARRIVE guidelines recommended for reporting animal research

Laboratory animals

Rhesus macaques, Chinese origin, adult (4-6 years), 6 males, 6 females

Wild animals

No wild animals were used. 
Field-collected samples

Ethics oversight

\section{No samples were collected in the field.}

All animal experiments were approved by the Institutional Animal Care and Use Committee of Rocky Mountain Laboratories, NIH and carried out by certified staff in an Association for Assessment and Accreditation of Laboratory Animal Care (AAALAC) International accredited facility, according to the institution's guidelines for animal use, following the guidelines and basic principles in the NIH Guide for the Care and Use of Laboratory Animals, the Animal Welfare Act, United States Department of Agriculture and the United States Public Health Service Policy on Humane Care and Use of Laboratory Animals.

Note that full information on the approval of the study protocol must also be provided in the manuscript. 